\title{
Optimization of a Third-Order Gradiometer for Operation in Unshielded Environments
}

\author{
S. A. Uzunbajakau, A. P. Rijpma, H. J. M. ter Brake, and M. J. Peters
}

\begin{abstract}
The optimum geometry of a third-order gradiometer for operation in unshielded environments is discussed. The optimization result depends on the specific signal and noise conditions. The fetal heart is considered as an example of the signal source. We optimized the gradiometer such that the signal-to-noise ratio is maximized in an averaged sense for all relevant environmental noise conditions and distances to the signal source. The resulting design consists of two second-order gradiometers that can be combined to form a third-order gradiometer in noisy environments, whereas a single second-order gradiometer can be used in lownoise environments. The gradiometer can provide the signal-tonoise ratio that allows detection of fetal heart signals in all relevant environmental noise conditions.
\end{abstract}

Index Terms-Biomagnetism, fetal magnetocardiography, gradiometer optimization.

\section{INTRODUCTION}

$\mathbf{T}$ HE gradiometer optimization was performed as a part of the FHARMON project. This project aims at the development of a single-channel fetal magnetocardiograph that can be operated unshielded in standard clinical environments. The very weak magnetic field of a fetal heart is measured by means of a low-Tc $(4.2 \mathrm{~K})$ superconducting quantum interference device (SQUID) [1]. The peak magnitude of fetal magnetocardiograms (fMCGs) equals $1 \mathrm{pT}$ on average. The spectral content of the signal is typically in between 1 and $100 \mathrm{~Hz}$. For a successful recording of fMCGs, a resolution better than $10 \mathrm{fT} / \sqrt{\mathrm{Hz}}$ is required [2]. In unshielded environments, this resolution is hard to achieve since the interference from environmental sources can have a spectral density up to $10 \mathrm{nT} / \sqrt{\mathrm{Hz}}$ at the frequency of $1 \mathrm{~Hz}$ [3], which is one million times the required sensitivity level.

Distant interference sources produce magnetic fields that are almost uniform in the region of the measurement site. In contrast, the magnetic field produced by the close-by fetal heart has relatively large higher order gradients. Discrimination of the fetal heart signal from the background environmental noise is achieved by configuring the magnetic field sensor such that it becomes insensitive to lower order spatial gradients of the magnetic field. Such a configuration of sensors is referred to as a gra-

Manuscript received April 18, 2005; revised June 13, 2005. This paper was recommended by Associate Editor M. Mueck. This work was supported by the Dutch Technology Foundation (STW), the Institute for Biomedical Technology (BMTI), Philips Medical Systems, Twente Medical Systems, and Thales Cryogenics.

The authors are with the Faculty of Science and Technology, University of Twente, 7500 AE Enschede, The Netherlands (e-mail: s.uzunbajakau@ utwente.nl; a.p.rijpma@tnw.utwente.nl; h.j.m.terbrake@tnw.utwente.nl; m.j.peters@tnw.utwente.nl).

Digital Object Identifier 10.1109/TASC.2005.854300
A)

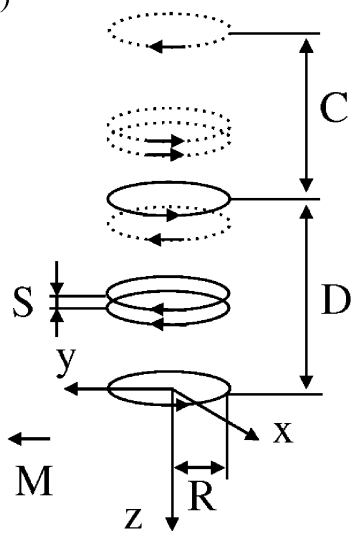

B)

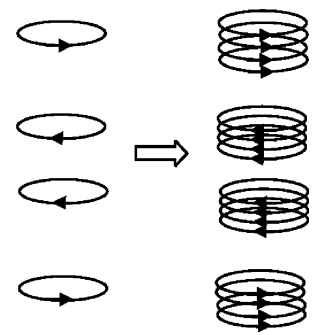

Fig. 1. A) The third-order gradiometer to be optimized. The gradiometer consists of two second-order gradiometers (solid and dashed lines). The second-order gradiometer (solid lines) will be used in low-noise environments while the third-order gradiometer will be used in noisy environments. A magnetic dipole $(M)$ is used to model the magnetic field generated by the fetal heart (see text). B) An illustration of the formation of a symmetric multiturn second-order gradiometer.

diometer. When operated outside a magnetically shielded room, a fetal magnetocardiograph requires a gradiometer of second or even third order [2], [4].

In this paper, we limit ourselves to the optimization of the third-order gradiometer that is shown in Fig. 1(a). The gradiometer consists of two second-order gradiometers. The signals of the two second-order gradiometers are read out separately and subtracted in the computer software. The order of the gradiometer can be adapted to the environmental noise level. That is, the second-order gradiometer can be used in low-noise environments whereas the third-order gradiometer can be used in relatively noisy environments.

The signal-to-noise ratio (SNR) depends on the radii of the sensing coils of the gradiometer and the distances between the coils. The optimal geometry of the gradiometer, i.e., the one that maximizes the SNR, depends on the level of environmental interference as well as on the position of the signal source in relation to the gradiometer. Different values of those parameters require gradiometers of different geometries. In our optimization, we determine the geometry of the gradiometer that provides the maximum of the mean SNR averaged for all combinations of the parameters. The magnetic noise that arises from the conducting parts of the measuring setup decreases the SNR as well. In our optimization, we deduce the maximum level of the magnetic noise that allows recording fMCG signals of all relevant magnitudes with a sufficient SNR. 
TABLE I

SUMmary OF THE ASSUMED VARIABLES THAT WERE USED TO CALCULATE THE SNR

\begin{tabular}{|c|c|c|}
\hline$\overline{\text { Variable }}$ & Description & Value \\
\hline$L_{i n}$ & $\begin{array}{l}\text { Input self inductance } \\
\text { of SQUID }[n H]\end{array}$ & 320 \\
\hline$M_{i n}$ & $\begin{array}{l}\text { Input mutual inductance } \\
\text { of SQUID }[n H]\end{array}$ & 10 \\
\hline$l$ & Length of the twisted wires $[\mathrm{mm}]$ & 300 \\
\hline$\Phi_{s}$ & $\begin{array}{l}\text { Equivalent flux noise } \\
\text { of SQUID }\left[\mu \Phi_{0} / \sqrt{H z}\right]\end{array}$ & 7.2 \\
\hline$B_{s}$ & $\begin{array}{l}\text { Field noise of the radiation } \\
\text { shield }[f T / \sqrt{H z}]\end{array}$ & $0 \ldots 10^{\star}$ \\
\hline$z$ & $\begin{array}{l}\text { Depth of the equivalent } \\
\text { dipole }[\mathrm{mm}]\end{array}$ & $50: 50: 150^{*}$ \\
\hline$M$ & $\begin{array}{l}\text { Magnitude of the equivalent } \\
\text { magnetic dipole }\left[n A \cdot m^{2}\right]\end{array}$ & 7 \\
\hline$G_{E N V}^{(2)}$ & $\begin{array}{l}\text { Second-order gradient of the } \\
\text { env. interference }\left[T \cdot \mathrm{m}^{-2}\right]\end{array}$ & $\begin{array}{c}0.53 \cdot 10^{-10} \\
\times \xi\end{array}$ \\
\hline$G_{E N V}^{(3)}$ & $\begin{array}{l}\text { Third-order gradient of the } \\
\text { env. interference }\left[T \cdot m^{-3}\right]\end{array}$ & $\begin{array}{c}0.11 \cdot 10^{-11} \\
\times \xi\end{array}$ \\
\hline$\xi$ & Noise parameter & $0 \ldots 1^{\star}$ \\
\hline$R$ & Radius of the gradiometer $[\mathrm{mm}]$ & $5: 2.5: 200^{*}$ \\
\hline$D$ & $\begin{array}{l}\text { Length of the second-order } \\
\text { gradiometers }[\mathrm{mm}]\end{array}$ & $20: 10: 300^{*}$ \\
\hline$S$ & $\begin{array}{l}\text { Separ. between the inner coils of the } \\
\text { second-order gradiometers }\end{array}$ & $\begin{array}{l}(1: 5.16: 99) \\
\times 10^{-2} \times D^{*}\end{array}$ \\
\hline $\bar{C}$ & $\begin{array}{l}\text { Separ. between the two second-order } \\
\text { gradiometers }[\mathrm{mm}]\end{array}$ & $10: 10: 300^{*}$ \\
\hline$m$ & $\begin{array}{l}\text { the number of turns in each coil } \\
\text { of the second-order gradiometer }\end{array}$ & $2: 1: 5 *$ \\
\hline$\triangle z$ & $\begin{array}{l}\text { the separation between the turns } \\
\text { of the second-order gradiometer }[\mathrm{mm}]\end{array}$ & $1 \ldots 20^{\star}$ \\
\hline
\end{tabular}

The second-order gradiometers that are shown in Fig. 1(a) have one turn of superconducting wire in each coil. Gradiometers that have several turns in each coil are frequently used in the instrumentation for biomagnetism. An example of such a multiturn gradiometer is shown in Fig. 1(b). The usefulness of multiturn gradiometers in our application is investigated as well.

\section{METHOD}

The gradiometer has four parameters that have to be optimized [see Fig. 1(a)]: the radius of the sensing coils $(R)$, the length of the second-order gradiometer $(D)$, the separation between the two inner turns of the second-order gradiometer $(S)$, and the separation between the two second-order gradiometers $(C)$.

First, the measuring system, the signal source, and the environmental noise are discussed (Sections II-A-II-C). Then, in Section II-D, the equation that was used to evaluate the SNR is given. The assumed values of the variables that were used to calculate the SNR are summarized in Table I. The optimization procedure is discussed in Section II-F, after the introduction of a performance function in Section II-E. Finally, in Section II-G, the method that was used to investigate the usefulness of the multiturn gradiometers in our application is discussed.

\section{A. Measuring System}

The standard layout of a SQUID measuring system is depicted in Fig. 2. The magnetic flux $\Phi_{g}$ through the gradiometer

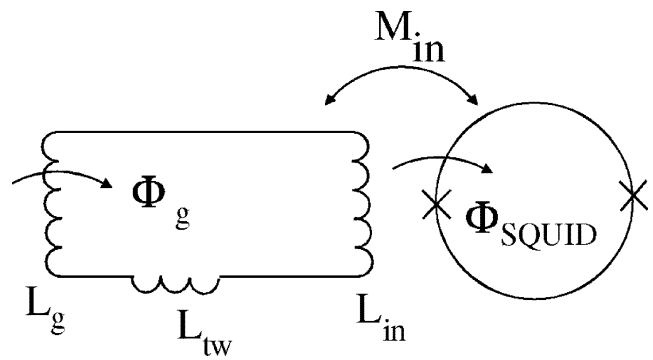

Fig. 2. Standard layout of a SQUID measuring system.

with inductance $L_{g}$ induces a current in the superconducting closed circuit $L_{g}, L_{t w}, L_{\text {in }}$. The current flowing in the input inductance $L_{\text {in }}$ produces a magnetic flux $\Phi_{\text {SQUID }}$ in the SQUID. The flux transfer efficiency equals

$$
K_{\Phi}=\frac{\Phi_{\mathrm{SQUID}}}{\Phi_{g}}=\frac{M_{\mathrm{in}}}{L_{\mathrm{in}}+L_{g}+L_{t w}}
$$

where $M_{\text {in }}$ is the mutual inductance between the input coil and the SQUID and $L_{t w}$ is the self inductance of the twisted wires that interconnect the coils of the gradiometer and connect them to the SQUID. The whole setup is enclosed by several layers of aluminum-coated Mylar film (superinsulation) as well as a radio-frequency interference (RFI) shield. The RFI shield is typically made of a single layer of a conducting material such as aluminum paint, aluminum foil, or copper meshing. Both the superinsulation and the RFI shield comprise conductive components and produce magnetic noise [5], [6]. The shielding by the RFI shield improves with increasing thickness and conductivity of the material that is used in the shield. In practice, it is desirable to have a shielding effect as large as possible. However, the magnetic noise due to the shield increases with the thickness and the conductivity of the material as well. A similar tradeoff holds for the superinsulation. Therefore, the maximum level of the magnetic noise due to the shield that allows realizing an adequate SNR needs to be investigated. In our optimization, we vary this magnetic noise $B_{s}$ between 0 and $10 \mathrm{fT} / \sqrt{\mathrm{Hz}}$, which is the worst sensitivity level that is acceptable in fetal magnetocardiography [2]. We optimize the geometry of the gradiometer for all assumed values of $B_{s}$ and then choose $B_{s}$ such that the SNR is acceptable.

The intrinsic sensitivity of the measuring setup is determined by two noise sources: the magnetic field noise of the RFI shield plus the superinsulation $\left(B_{s}\right)$ and the magnetic flux noise of the SQUID plus readout electronics $\left(\Phi_{s}\right)$. In the FHARMON project demonstrator, we will use the commercially available SQUIDs type CSblue of Supracon [7]. The parameters of the SQUIDs are

$$
\Phi_{s}=7.2 \frac{\mu \Phi_{0}}{\sqrt{\mathrm{Hz}}} ; L_{\mathrm{in}}=320 \mathrm{nH} ; M_{\mathrm{in}}=10 \mathrm{nH} .
$$

The self inductance of the gradiometer was evaluated using expressions that are available in [8]. The self inductance of the twisted wires $\left(L_{t w}\right)$ was evaluated using the following expression [9]:

$$
L_{t w}=0.5 l
$$


where $l$ is the length of the twisted wires in millimeters and $L_{t w}$ is the resulting inductance in nanohenries.

\section{B. Signal Source}

The magnetic field due to the fetal heart plus the field due to the volume currents can be modeled by an equivalent magnetic dipole [10]. The position of the dipole in relation to the gradiometer is shown in Fig. 1(a). The gradiometer is invariant to the rotation around the $z$-axis. Consequently, we choose the coordinate system such that the $x$-component of the displacement vector equals zero. The $x$-component of the equivalent magnetic dipole does not contribute to the $z$-component of the magnetic field and is omitted from the consideration. The results presented in [10] suggest that the angle between the equivalent magnetic dipole and the $x y$-plane is at most $40^{\circ}$ where the $x y$-plane is tangential to the maternal abdominal surface. Our calculations show that a rotation of the magnetic dipole out of the $x y$-plane by $40^{\circ}$ does not affect the optimization results significantly. Therefore, the $z$-component of the equivalent magnetic dipole is neglected.

The net flux through the gradiometer due to the equivalent magnetic dipole was evaluated by integrating numerically the following equation:

$$
\Phi_{D}=\left|\sum_{m=1}^{N} s_{m} \frac{\mu_{0}}{4 \pi} \int_{0}^{2 \pi} \frac{R \cdot M \cdot\left(z-z_{m}\right) \cdot \sin \beta \cdot d \beta}{\left[(R \cos \beta)^{2}+(y-R \sin \beta)^{2}+\left(z-z_{m}\right)^{2}\right]^{3 / 2}}\right|
$$

where $z_{m}$ stands for the $z$-coordinate of the $m$ th turn of the gradiometer and $s_{m}=1$ if the $m$ th turn of the gradiometer is wound clockwise; $s_{m}=-1$ if the turn is wound counterclockwise; $N$ is the number of turns in the gradiometer; and $\beta$ is the parameter of the parameterization of a single turn. The depth of the dipole was assumed to be equal to $z=0.05,0.1$, and $0.15 \mathrm{~m}$. These correspond to the minimum, the medium, and the maximum depths expected for the equivalent magnetic dipole. The $y$-coordinate of the dipole was chosen such that the net magnetic flux through the gradiometer was maximized. The minimal expected value of the magnitude of the equivalent magnetic dipole

$$
M=7 \mathrm{nA} \cdot \mathrm{m}^{2}
$$

was estimated based on data presented in [11].

\section{Environmental Interference}

The maximal expected magnitudes of power spectral density (PSD) at the frequency of $1 \mathrm{~Hz}$ of the second- and third-order gradients of the interfering environmental magnetic field were estimated from the collection of PSDs of the zero- and firstorder gradients presented in [3]. In order to make this estimation, it was assumed that the magnitude of the interfering magnetic fields is proportional to the inverse cube of the distance. Based on the estimated magnitudes and assuming a worst-case frequency dependence of the PSD to be proportional to $1 / f$, the maximal root mean square (rms) values of the second- and third-order gradients of the interfering magnetic field were determined as

$$
\begin{aligned}
& G_{\mathrm{MAX}}^{(2)}=0.53 \cdot 10^{-10} \mathrm{~T} \cdot \mathrm{m}^{-2} \\
& G_{\mathrm{MAX}}^{(3)}=0.11 \cdot 10^{-11} \mathrm{~T} \cdot \mathrm{m}^{-3} .
\end{aligned}
$$

Here, the frequency content of the fMCG signal was taken to be between 1 and $100 \mathrm{~Hz}$. In our optimization, we use an environmental noise parameter $\xi$ between 0 and 1 such that the actual rms value of a gradient of the interfering magnetic field that is considered is

$$
G_{\mathrm{ENV}}^{(n)}=G_{\mathrm{MAX}}^{(n)} \cdot \xi
$$

where $n$ is the order of the gradient. The second- and thirdorder gradiometers are assumed to be sensitive to the secondand third-order gradients of the interfering magnetic field only. That is, the gradiometers are assumed to be perfectly balanced and the higher order gradients of the interfering magnetic field are neglected. The net flux through the gradiometer due to the environmental interference was evaluated as

$$
\Phi_{\mathrm{ENV}}^{(n)}=G_{\mathrm{ENV}}^{(n)} \cdot b^{(n)}
$$

where $b^{(n)}$ is the sensitivity of the gradiometer to the corresponding gradient. Neglecting the spatial variations of the interfering magnetic field in the transversal directions, these sensitivities can be evaluated as

$$
\begin{aligned}
& b^{(2)}=0.5 \cdot \pi R^{2} \cdot\left(D^{2}-S^{2}\right) \\
& b^{(3)}=3 \cdot C \cdot b^{(2)}
\end{aligned}
$$

where $R, D, S$, and $C$ are the geometrical parameters described by Fig. 1(a).

Apart from the intentional sensitivities given in (10) and (11), a practical gradiometer has parasitic sensitivities (so-called imbalances) to lower order gradients of the interfering magnetic field [12]. However, we assume that the second- and third-order gradiometers are manufactured and balanced sufficiently well to be in their intrinsic regimes [12]. In that case, the noise component of the signal is defined either by the intrinsic noise of the measuring system or by the second- or third-order gradients of the interfering magnetic field. The field imbalance that is required to keep the gradiometers designed in the subsequent sections in their intrinsic regimes is of order of $10^{-6}$. The required first-order gradient imbalance is of the order of $10^{-3}-10^{-4}$. From [12], it was concluded that it is feasible to realize these imbalances using electronic balancing. Consequently, imbalance contributions are neglected in our optimization procedure.

\section{Signal-to-Noise Ratio}

The SNR of the fMCGs is expressed as

$$
\begin{aligned}
& \mathrm{SNR}=20 \\
& \times \log \frac{\Phi_{D}}{\sqrt{\left(\Phi_{\mathrm{ENV}}^{(n)}\right)^{2}+\triangle f\left(\pi R^{2} B_{s}\right)^{2}+(n-1) \triangle f \frac{\left(\Phi_{s}\right)^{2}}{K_{\Phi}^{2}}}}
\end{aligned}
$$


where $\triangle f=100 \mathrm{~Hz}$ is the bandwidth of the fetal heart signal. The numerator in the last equation equals the amplitude of the fetal heart signal expressed as the net magnetic flux through the gradiometer. The denominator equals the rms value of the interfering noise expressed as the net magnetic flux through the gradiometer as well. The three terms in the denominator represent the rms values of the environmental noise, the magnetic noise of the RFI shield plus the superinsulation, and the noise of SQUID plus readout electronics. In order to determine the required SNR, a noiseless fMCG signal was mixed in different proportions with white noise that represented the noise of the measuring system. Based on the resultant mixtures of the $\mathrm{fMCG}$ signal and noise, it was concluded that a SNR of $15 \mathrm{~dB}$ is required in order to make the fetal heart beats discernible in the fMCG recordings (see Section III-A).

\section{E. Optimization Performance Function}

The SNR given in (12) can be viewed as a function of seven independent variables

$$
\mathrm{SNR}=\operatorname{SNR}\left(D, R, S, C, B_{s}, z, \xi\right) .
$$

These independent variables can be divided in two groups. The first group comprises the geometrical parameters of the gradiometer $(D, R, S, C)$ that have to be optimized. The second group comprises the parameters of the environment in which the gradiometer is operated $\left(B_{s}, z, \xi\right)$. The parameters $z$ and $\xi$ are independent of the optimization procedure. The parameter $B_{s}$ is determined by optimizing the geometry of the gradiometer for all assumed values of $B_{s}$ and choosing $B_{s}$ such that the SNR is acceptable.

The performance function is introduced by means of a simplified example in which the SNR is assumed to be a function of one geometrical parameter of the gradiometer $\left(P_{g}\right)$ and one parameter of the environment $\left(P_{e}\right)$

$$
\operatorname{SNR}=\operatorname{SNR}\left(P_{g}, P_{e}\right)
$$

For instance, the parameter $P_{g}$ could represent the radius of the gradiometer $(R)$ and the parameter $P_{e}$ could represent the depth of the equivalent magnetic dipole $(z)$. In Fig. 3, schematic curves of the SNR versus the parameter of the gradiometer are shown. The two solid curves represent the SNR of the gradiometers that operate in different environments that are described by $P_{e}=P_{e 1}$ and $P_{e}=P_{e 2}$. If the parameter of the gradiometer is chosen to be $P_{g 0}$, then the actual SNR is $d_{1}$ lower than the maximal obtainable in the case $P_{e}=P_{e 1}$ and $d_{2}$ below the maximal SNR in the case $P_{e}=P_{e 2}$. The optimization of $P_{g}$ aims at a maximum of the averaged SNR indicated in Fig. 3. Therefore, the performance function can be expressed as

$$
J\left(P_{g}\right)=\frac{1}{2}\left(\operatorname{SNR}\left(P_{g}, P_{e 1}\right)+\operatorname{SNR}\left(P_{g}, P_{e 2}\right)\right) .
$$

The performance function given by the last equation differs from the actual performance function used in our optimization in the number of the parameters considered.

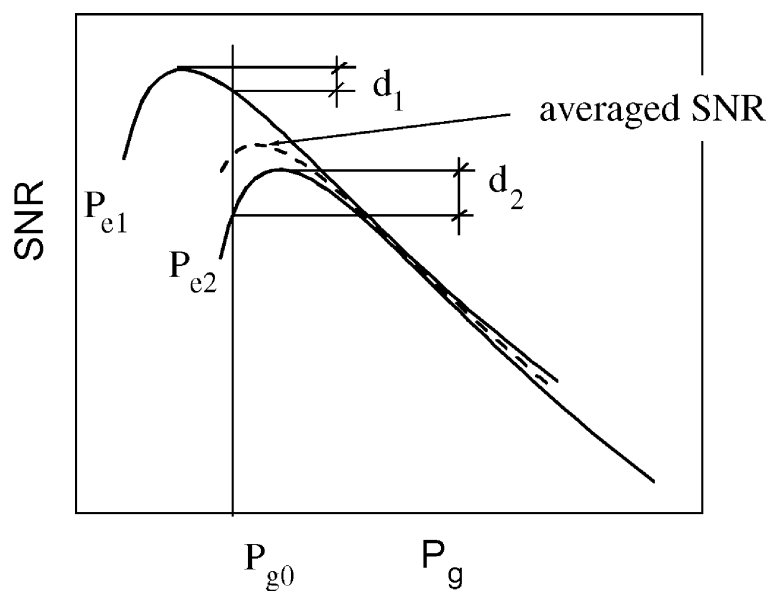

Fig. 3. A stylized plot that illustrates the optimization concept. The two solid curves correspond to gradiometers that operate in two different environments that are described by parameters $P_{e 1}$ and $P_{e 2}$. If the parameter of the gradiometer is chosen to be equal to $P_{g 0}$, the SNRs will deviate from their maxima by $d_{1}$ and $d_{2}$, respectively. The optimization aims at the maximization of the averaged SNR.

\section{F. Optimization Procedure}

The optimization consists of two steps. In the first step, the optimal geometrical parameters of the second-order gradiometer are derived. Equation (12) is evaluated using the set of values of variables listed in Table I. Consequently, for each combination $(i)$ of the environmental conditions, the following function of three variables is derived:

$$
\mathrm{SNR}_{i}=\mathrm{SNR}_{i}(D, R, S) .
$$

The mean SNR [similar to (15)] averaged for different combinations is evaluated as

$$
J^{(2)}(D, R, S)=\frac{1}{N} \sum_{i=1}^{N} \operatorname{SNR}_{i}(D, R, S)
$$

where $N$ is the number of combinations of the environmental parameters. The arguments of the maximum of the last equation are considered as the geometrical parameters of the optimal second-order gradiometer.

The third-order gradiometer consists of two identical second-order gradiometers that are optimized as discussed above. In the second step of the optimization, the axial separation $(C)$ between the two second-order gradiometers is derived following the same procedure that is used for the optimization of the second-order gradiometer with the difference that the performance function has only one independent variable

$$
J^{(3)}(C)=\frac{1}{N} \sum_{i=1}^{N} \operatorname{SNR}_{i}(C) .
$$

\section{G. Number of Turns}

The number of turns of the gradiometer coils has a two-fold effect on the signal transferred to the SQUID. First, it increases the net magnetic flux through the gradiometer which roughly 


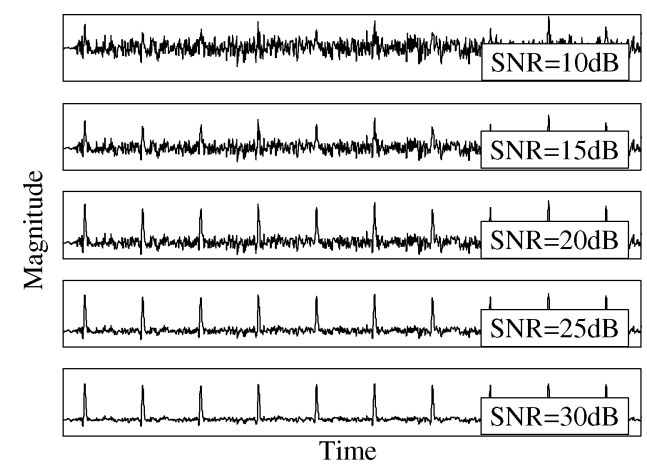

Fig. 4. A noiseless fMCG signal mixed in different proportions with white noise. This figure is used for the qualitative evaluation of the SNR. It was concluded that a SNR of $15 \mathrm{~dB}$ is sufficient to make the fetal heart beats discernible in $\mathrm{fMCG}$ recordings.

scales with the number of turns. Second, it decreases the flux transfer efficiency which is given by (1) because the self inductance of the gradiometer $L_{g}$ increases with the number of turns as well. The inductance of the gradiometer increases more rapidly with the number of turns than the net magnetic flux through the gradiometer does. Therefore, the signal transferred to the SQUID can be increased by increasing the number of turns only if the self inductance of the SQUID input coil is sufficiently large compared to that of the gradiometer.

A one-turn and a corresponding four-turn second-order gradiometers are shown in Fig. 1(b) as an example. The usefulness of the $m$-turn gradiometers is investigated by comparing the magnetic flux transferred to the SQUID in the case of a one-turn gradiometer with that of an $m$-turn gradiometer

$$
Q=20 \log \left(\frac{\Phi_{m}}{L_{m}+L_{\text {in }}+L_{t w}} \frac{L_{1}+L_{\text {in }}+L_{t w}}{\Phi_{1}}\right)
$$

where $L_{1}$ and $L_{m}$ are the self inductances of the one-turn and the corresponding $m$-turn gradiometers and $\Phi_{1}$ and $\Phi_{m}$ the net magnetic fluxes, respectively. Increasing the number of turns increases the magnetic flux coupled to the SQUID only if the parameter $Q$ given by the last equation is greater than zero.

\section{RESULTS}

\section{A. Signal-to-Noise-Ratio}

The fMCG signal mixed with white noise in different proportions is shown in Fig. 4. From these plots, it was concluded that the SNR of the fetal magnetocardiogram has to be in the range $10-15 \mathrm{~dB}$ in order to make the fetal heart beats discernible in the recordings. This would allow the averaging of the fetal heart beats with the subsequent determination of the intracardiac intervals [10]. In the case of the third-order gradiometer, the contribution of the SQUIDs to the noise power of the measuring system is twice as large as in the case of second-order gradiometer. Assuming that the third-order gradiometer rejects all environmental interferences, the difference in the SNR between the second-order gradiometer under condition $\xi=0$ and the third-order gradiometer for all values of $\xi$ is less than $3 \mathrm{~dB}$. Thus, the SNR of the second-order gradiometer in a noise-free

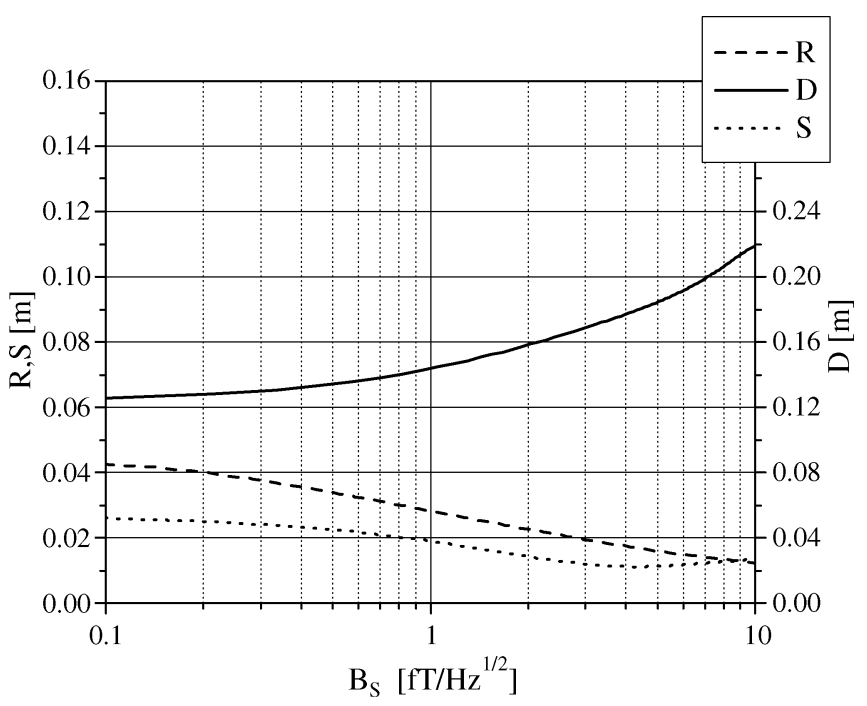

Fig. 5. Optimal length $(D)$, the radius $(R)$, and the separation between the inner coils $(S)$ versus magnetic noise $B_{s}$. The environmental noise parameter is assumed to be in between $\xi=10^{-4}$ and $10^{-1}$. Other parameters are assumed to have values that are listed in Table I.

environment $(\xi=0)$ could be considered as a "SNR characteristic" of both second- and third-order gradiometers. In what follows, we consider the gradiometer to be designed sufficiently well if the SNR of the weakest fMCG signal measured by the second-order gradiometer in the noise-free environment is equal to $15 \mathrm{~dB}$. The weakest $\mathrm{fMCG}$ signal considered corresponds to the smallest magnitude of the equivalent magnetic dipole of $7 \mathrm{nA} \cdot \mathrm{m}^{2}$ and the largest depth of the dipole of $0.15 \mathrm{~m}$.

\section{B. Optimization of the Second-Order Gradiometer}

In Fig. 5, the radius, the length, and the separation between the inner turns of the single-turn second-order gradiometers that are optimal for $\xi=10^{-4}-10^{-1}$ and $z=0.05,0.1,0.15 \mathrm{~m}$ are shown as a function of $B_{s}$. The parameter $\xi$ was varied with a unitary step in the exponent. The lower limit of the parameter $\xi=10^{-4}$ is chosen such that the contribution of the environmental interference to the SNR can be neglected. The curves that are presented in Fig. 5 are the results of polynomial fits of the calculated data. These fits were made to smoothen quantization errors due to the finite step in the grid of values of independent variables for which (12) was evaluated. In the absence of the magnetic noise of the RFI shield and the superinsulation ( $B_{s}=0 \mathrm{fT} / \sqrt{\mathrm{Hz}}$; not shown in Fig. 5), the optimal gradiometer has the largest radius $R=0.045 \mathrm{~m}$ and the shortest length $D=0.12 \mathrm{~m}$. The increase of the magnetic noise leads to an increase of the gradiometer length and a decrease of its radius.

In Fig. 6, the SNR, calculated for the gradiometers of the optimal dimensions that are presented in Fig. 5, is shown. The SNR was calculated assuming a noise-free environment $(\xi=0)$. From Fig. 6, it follows that, if the magnetic noise due to the RFI shield and the superinsulation equals $1.5 \mathrm{fT} / \sqrt{\mathrm{Hz}}$, the recording of the weakest fMCG signal will have a SNR of $15 \mathrm{~dB}$ which is sufficient for the detection of the fetal heart beats. The geometrical parameters of the corresponding gradiometer can be deduced from Fig. 5 as $R=0.025 \mathrm{~m}, D=0.15 \mathrm{~m}, S=0.016 \mathrm{~m}$. This gradiometer in combination with a maximum shield noise 


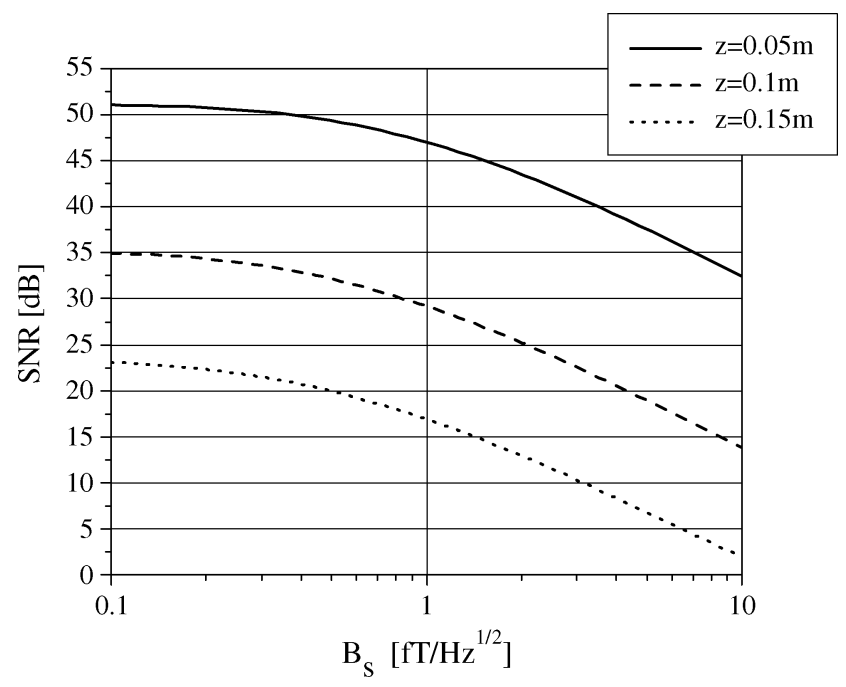

Fig. 6. SNR calculated for the second-order gradiometers of the optimal dimensions that are shown in Fig. 5. The environmental noise parameter is assumed to be equal to $\xi=0$. The magnitude of the equivalent dipole is assumed to be equal to the minimal expected value.

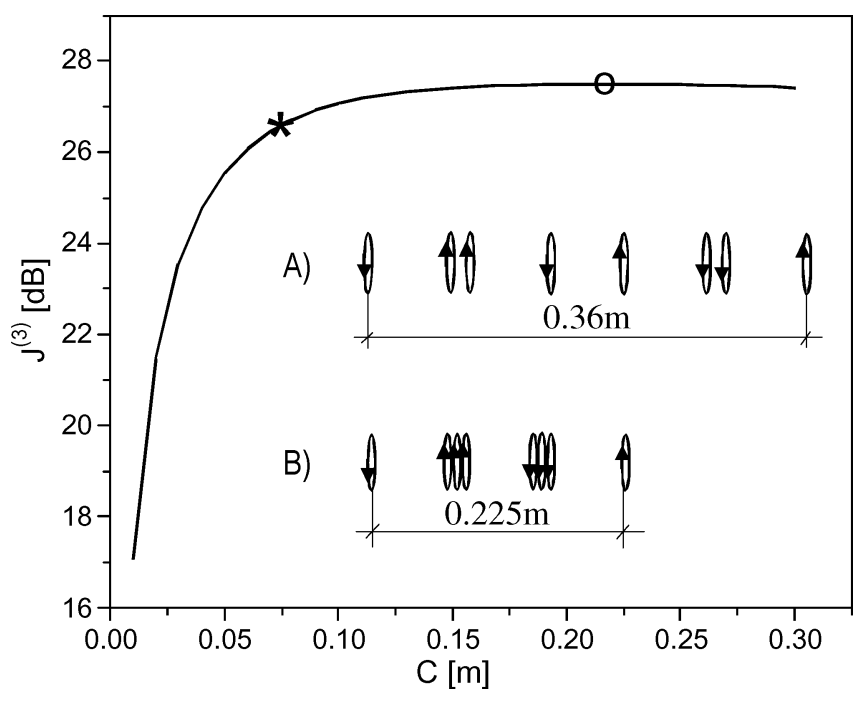

Fig. 7. Performance function of the optimization of the third-order gradiometer $[(18)$ in the text]. The maximum of the performance function (indicated by the circle) corresponds to the separation between the second-order gradiometers of $C=0.21 \mathrm{~m}$. The decrease of the separation to a value of $0.075 \mathrm{~m}$ (indicated by the asterisk) leads to a decrease of the performance function by $0.8 \mathrm{~dB}$. The third-order gradiometers that correspond to the separations of $C=0.21 \mathrm{~m}$ and $C=0.075 \mathrm{~m}$ are shown in insets $\mathrm{A}$ ) and $\mathrm{B}$ ), respectively.

$B_{s}$ of $1.5 \mathrm{fT} / \sqrt{\mathrm{Hz}}$ provides an adequate performance of the gradiometer. A better performance in terms of SNR can only be obtained if $B_{s}$ can be reduced to a lower level. Then the geometry of the gradiometer would change as indicated in Fig. 5.

\section{Optimization of the Third-Order Gradiometer}

The performance function (18) for the optimization of the third-order gradiometer is shown in Fig. 7. For the noise parameter $(\xi), 20$ points logarithmically spaced between 0.1 and 1 were taken. The shield noise and the geometrical parameters of the two second-order gradiometers are assumed to be equal to the optimal ones discussed above. The maximum of the performance function is designated in Fig. 7 by the circle. The

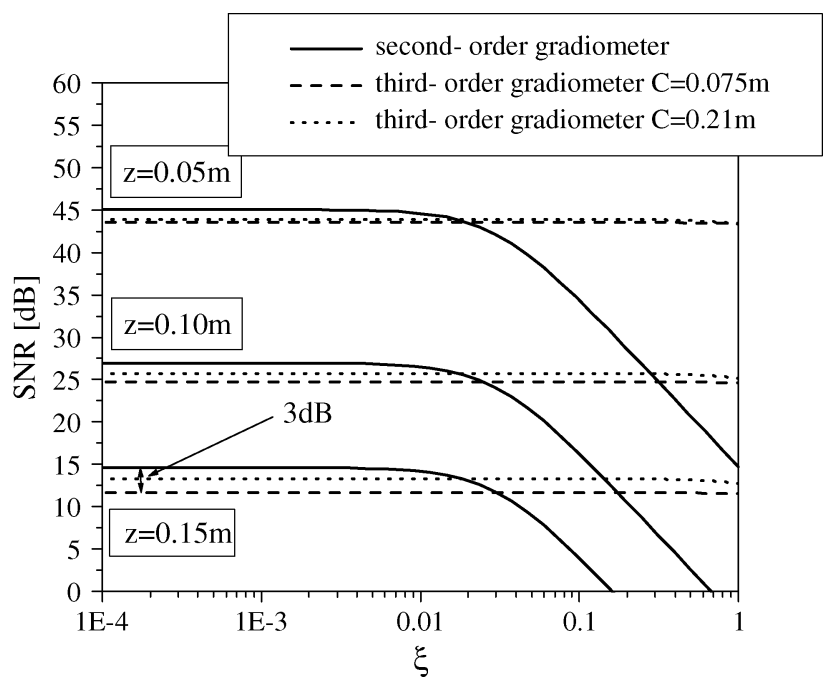

Fig. 8. SNR curves calculated for the second- and third-order gradiometers assuming $B_{s}=1.5 \mathrm{fT} / \sqrt{\mathrm{Hz}}$ and the minimal magnitude of the equivalent magnetic dipole.

maximum corresponds to a separation between the second-order gradiometers of $0.21 \mathrm{~m}$. However, a decrease of the separation from 0.21 to $0.075 \mathrm{~m}$ (indicated in Fig. 7 by an asterisk) leads to a small decrease of the performance function from 27.5 to $26.7 \mathrm{~dB}$. The two third-order gradiometers that correspond to the separations of 0.21 and $0.075 \mathrm{~m}$ are shown in Fig. 7 as well. The decrease of the separation between the two secondorder gradiometers leads to a decrease of the total length of the third-order gradiometer of $37 \%$ without significant change of the performance. Therefore, in our design we choose a separation of $C=0.075 \mathrm{~m}$. The SNRs of the second- and third-order gradiometers are shown in Fig. 8 as a function of the environmental noise parameter. From the figure, it follows that the second-order gradiometer provides an improvement of the SNR up to $3 \mathrm{~dB}$ over the third-order gradiometer in the low-noise environments. The point where the SNR curves of the second- and third-order gradiometers intersect depends on the depth of the source and is around $\xi=0.02$. The second-order gradiometer will be used in environments that correspond to $\xi$ less than the above-mentioned point. The third-order gradiometer will be used otherwise.

\section{Number of Turns}

The optimal geometries of the one-turn second-order gradiometers are shown in Fig. 5 as a function of the magnetic noise of the RFI shield plus the superinsulation $\left(B_{s}\right)$. The possibility to increase the magnetic flux transferred to the SQUID by increasing the number of turns of the coils of the optimal second-order gradiometers is investigated by evaluating (19) for different optimal gradiometer geometries that are given in Fig. 5. The number of turns in each coil $(m)$, the separation between the turns $(\triangle z)$, and the depth of the equivalent magnetic dipole $(z)$ were varied as indicated in Table I. The parameter $Q$ calculated for $z=0.05 \mathrm{~m}$ and $B_{s}=0 \mathrm{fT} / \sqrt{\mathrm{Hz}}$, $B_{s}=1.5 \mathrm{fT} / \sqrt{\mathrm{Hz}}, B_{s}=10 \mathrm{fT} / \sqrt{\mathrm{Hz}}$ is shown in Fig. 9. The maximal values of the parameter $Q$ for the three gradiometer geometries are indicated in Fig. 9 by circles. The maximal values 


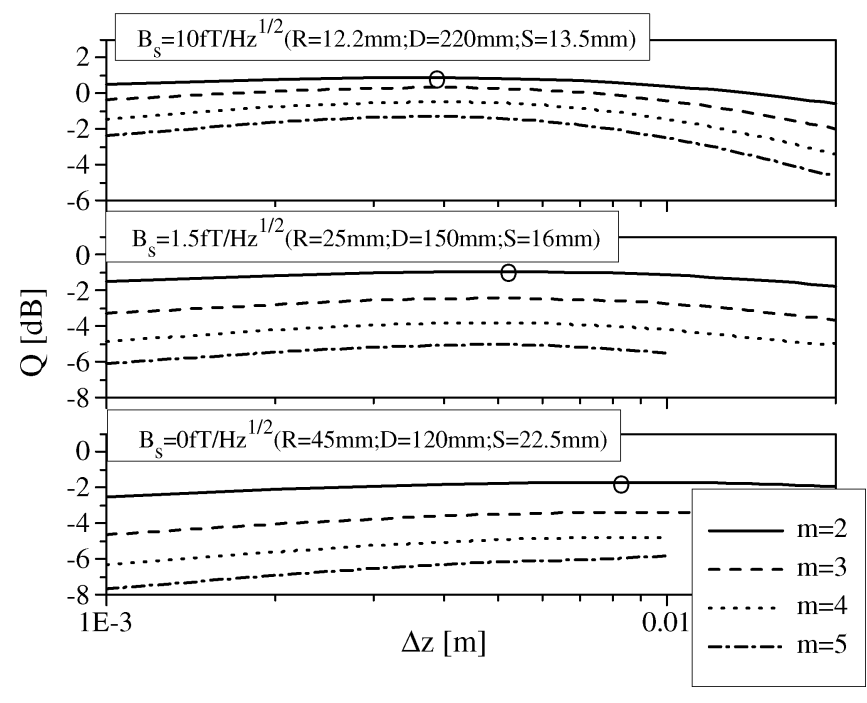

Fig. 9. Increase of the magnetic flux coupled to the SQUID [(19) in the text] due to increase of the number of turns of the gradiometer $(m)$ as a function of the separation between the turns $(\triangle z)$ with source depth $z=0.05 \mathrm{~m}$. The three plots correspond to three different geometries of the initial one-turn second-order gradiometer (see Fig. 5).

of the parameter $Q$ for other gradiometer geometries of Fig. 5 are shown in Fig. 10 as a function of the gradiometer radius. From this figure, it follows that the increase of the number of turns only makes sense if the radius of the gradiometer is less than about $20 \mathrm{~mm}$. This corresponds to a magnetic shield noise $B_{s}>3 \mathrm{fT} / \sqrt{\mathrm{Hz}}$ in Fig. 5 or minimal SNR $<10 \mathrm{~dB}$ in Fig. 6. Consequently, we conclude that the self inductance of the optimal gradiometer geometry that was discussed in the previous sections is too large to increase the magnetic flux transferred to the SQUID by increasing the number of turns $(R=0.025 \mathrm{~m}$, $D=0.15 \mathrm{~m}, S=0.016 \mathrm{~m}$ ).

\section{CONCLUSION}

A third-order gradiometer that consists of two identical symmetric second-order gradiometers was optimized for application in unshielded environments. A performance function was defined in which the average SNR is determined over a range of environmental noise conditions and distances to the signal source. This function was maximized in order to find the optimal gradiometer design. Fetal magnetocardiography was considered as an example of application. However, the approach to the optimization can be used in other applications as well. The optimized gradiometer is shown in Fig. 7(a). The geometrical parameters of the gradiometer are $R=0.025 \mathrm{~m}, D=0.15 \mathrm{~m}$, $S=0.016 \mathrm{~m}$, and $C=0.21 \mathrm{~m}$. It was found that the separation between the second-order gradiometers $(C)$ can be decreased without significant change in the SNR. The maximal magnetic noise due to the radio-frequency interference shield and the superinsulation that allows recording of the fMCG signals of the minimal magnitude with the sufficient SNR of $15 \mathrm{~dB}$ was found to be equal to $1.5 \mathrm{fT} / \sqrt{\mathrm{Hz}}$. The benefit of increasing the number of turns was investigated as well. In our specific example, the self inductance of the optimized gradiometer is too large for this increase to be advantageous.

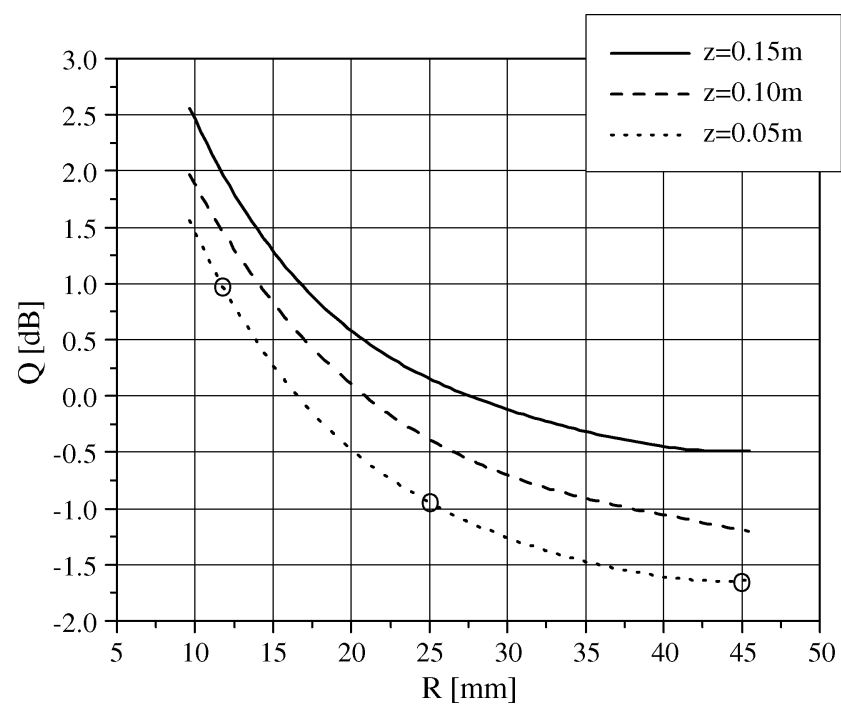

Fig. 10. Maximum possible increase of the magnetic flux coupled to the SQUID due to increase of the number of turns of the gradiometer. The circles in this figure correspond to the circles in Fig. 9.

\section{REFERENCES}

[1] J. Clarke, "SQUID fundamentals," in SQUID Sensors: Fundamentals, Fabrication and Applications, ser. NATO ASI Series E: Applied Sciences. Norwell, MA: Kluwer, 1996, vol. 329, pp. 1-62.

[2] H. J. M. ter Brake, A. P. Rijpma, J. G. Stinstra, J. Borgmann, H. J. Holland, H. J. G. Krooshoop, M. J. Peters, J. Flokstra, and H. W. P. Quartero, "Fetal magnetocardiography: Clinical relevance and feasibility," Physica C, vol. 368, pp. 10-17, 2002.

[3] J. Vrba and J. Mckay, "Character and acquisition of multichannel biomagnetic data," Appl. Supercond., vol. 5, pp. 431-439, 1998.

[4] S. E. Robinson, M. B. Burbank, A. A. Fife, G. Haid, P. R. Kubik, I Seachev, B. Taylor, B. Tillitoson, J. Vrba, G. Wong, C. L. Lowery, H. Eswaran, J. D. Wilson, P. Murphy, and H. A. Preissl, "Instrument for human reproductive assessment," in Biomag 2000, Proceedings of the 12th International Conference in Biomagnetism, H. A. Nenonen, Ed. Espoo, Finland: Helsinki Univ. Technol., 2001, pp. 919-922.

[5] A. P. Rijpma, "Fetal heart monitor: Development of a cryocooler-cooled high-Tc SQUID system for fetal magnetocardiography in unshielded environment," Ph.D. dissertation, Univ. Twente, The Netherlands, 2002.

[6] S. Uzunbajakau, A. P. Rijpma, J. Dolfsma, H. J. G. Krooshoop, H. J. M. ter Brake, M. J. Peters, and H. Rogalla, "Magnetic flux fluctuations due to eddy currents and thermal noise in metallic disks," IEEE Trans. Magn., vol. 39, no. 4, pp. 2018-2023, Jul. 2003.

[7] Supracon. [Online] http://www.supracon.com/

[8] H. J. M. ter Brake, "SQUID magnetometers," Ph.D. dissertation, Univ. Twente, The Netherlands, 1996.

[9] R. Cantor, "DC SQUIDS: Design, optimization and practical applications," in SQUID Sensors: Fundamentals, Fabrication and Applications, ser. NATO ASI Series E: Applied Sciences. Norwell, MA: Kluwer, 1996, vol. 329, pp. 179-233.

[10] J. G. Stinstra, "The reliability of the fetal magnetocardiogram," Ph.D. dissertation, Univ. Twente, The Netherlands, 2001.

[11] A. Kandori, T. Miyashita, K. Tsukada, H. Horigome, M. Asaka, S. Shigemitsu, M. I. Takahashi, Y. Terada, and T. Mitsui, "Sensitivity of foetal cardiograms versus gestation week," Med. Bio. Eng. Comput., pp. 545-548, 1999.

[12] J. Vrba, "SQUID gradiometers in real environments," in SQUID Sensors: Fundamentals, Fabrication and Applications, ser. NATO ASI Series E: Applied Sciences. Norwell, MA: Kluwer, 1996, vol. 329, pp. $117-178$.

Photographs and biographies of the authors were not available at the time of publication. 